\title{
The dynamic game theory methods applied to ship control with minimum risk of collision
}

\author{
J. Lisowski \\ Department of Ship Automation, Gdynia Maritime University, Poland
}

\begin{abstract}
This paper describes the application of elements of the game theory for the purpose of optimal control of some dynamic continuous processes. Using as an example the process of safe ship's control, the paper presents the problem of applying a positional non-cooperative game of $j$ objects for the description of the process considered as well as for the synthesis of optimal strategies. A mathematical model of differential game is formulated and its approximation in the form of a dual linear programming problem is used for the synthesis of safe ship's trajectory as a multistage process decision. The considerations have been illustrated in an example of a computer simulation (the POSGAME programme) to determine the safe ship's trajectory in a situation of passing many of the objects encountered.
\end{abstract}

Keywords: differential games, positional games, dual linear programming, safety navigation, risk analysis, ship control.

\section{Introduction}

In order to ensure safe navigation the ships are obliged to observe legal requirements contained in the International Regulations for Preventing Collision at Sea (COLREG). However, these Rules refer exclusively to two ships under good visibility conditions, in case of restricted visibility the Rules provide only recommendations of general nature and they are unable to consider all necessary conditions of the real process. Therefore the real process of the ships passing exercises occurs under the conditions of indefiniteness and conflict accompanied by an imprecise co-operation among the ships in the light of the legal regulations. Consequently, it is reasonable - for ship operational purposes - to present this process and to develop and examine methods for a safe steering of 
the ship by applying the rules of the game theory. A necessity to consider simultaneously the strategies of the encountered objects and the dynamic properties of the ships as the steering objects is a good reason for the application of the differential game model - often called the dynamic game - for the description of the processes $[1,10,12,13]$.

The challenge in research for effective methods to prevent ship collisions has become important with the increasing size, speed and number of ships participating in sea carriage. An obvious contribution in increasing safety of shipping has been firstly the application of radars and then the development of ARPA (Automatic Radar Plotting Aids) anti-collision system. The ARPA system enables to track automatically at least 20 encountered $j$ objects, determination of their movement parameters (speed $V_{j}$, course $\psi_{j}$ ) and elements of approach to the own ship $\left(D_{\min }^{j}=D C P A_{j}\right.$ - Distance of the Closest Point of Approach, $T_{\text {min }}^{j}=T C P A_{j}$ - Time to the Closest Point of Approach) and also the assessment of the collision risk $r_{j}$, fig. 1 .

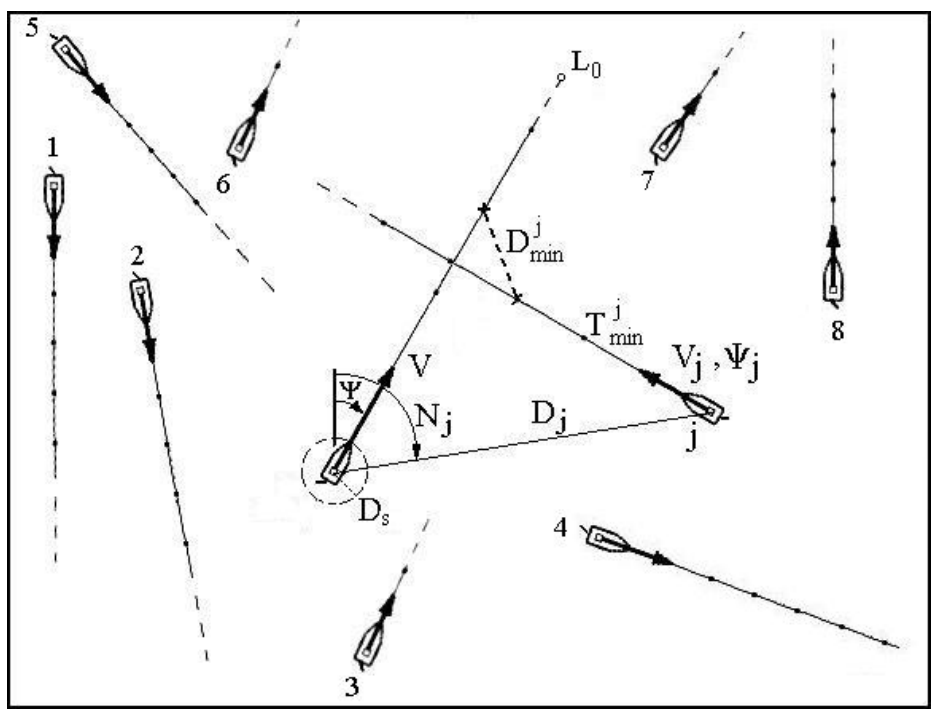

Figure 1: Navigational situation representing the passing of the own ship with the $j$-th object.

However the functional scope of a standard ARPA system ends with the simulation of the manoeuvre altering the course $\pm \Delta \psi$ or the ship's speed $\pm \Delta V$ selected by the navigator.

The most general description of the own control object passing the $j$ number of other encountered moving objects is the model of a dynamic game of a $j$ number of objects $[2,3,4,5,11]$. 


\section{The positional game model of process}

The dynamic game is reduced to a positional multistage game of a $j$ number of participants who do not co-operate among them [6, 7], fig. 2.

The state and control variables are represented by the following values:

$$
\left.\begin{array}{l}
x_{0}^{1}=X_{0}, x_{0}^{2}=Y_{0}, x_{j}^{1}=X_{j}, x_{j}^{2}=Y_{j}, u_{0}^{1}=\psi, u_{0}^{2}=V, u_{j}^{1}=\psi_{j}, u_{j}^{2}=V_{j} \\
j=1,2, \ldots, m
\end{array}\right\}
$$

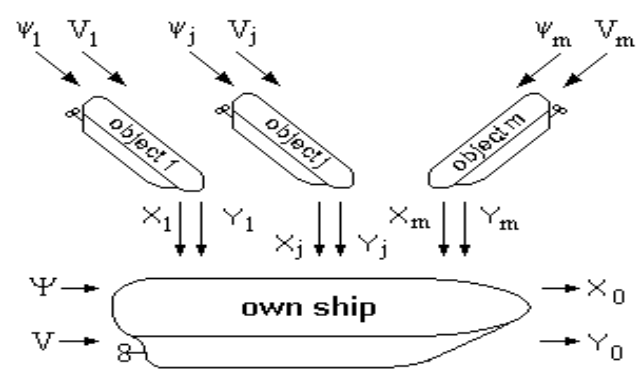

Figure 2: Block diagram of a positional model of the game.

The making of a continuous positional game discrete and reducing it to a multistage positional game is determined by own ship and depends on: the maximum relative speed of the own ship under the current navigational situation, the range of the situation and the dynamic characteristics of the ship [8].

The essence of the positional game is to make the strategies of the own ship dependent on current positions $p\left(t_{k}\right)$ of the objects encountered at the current step $k$. In this way possible course and speed alterations of the objects encountered are considered in the process model during the steering performance. The current state of the process is determined by the co-ordinates for the position of the own ship and of the objects encountered:

$$
x_{0}=\left(X_{0}, Y_{0}\right), x_{j}=\left(X_{j}, Y_{j}\right), j=1,2, \ldots, m
$$

The system generates its steering at the moment $t_{k}$ on the basis of the data which are obtained from the ARPA anti-collision system concerning the current positions of the ships:

$$
p\left(t_{k}\right)=\left[\begin{array}{l}
x_{0}\left(t_{k}\right) \\
x_{j}\left(t_{k}\right)
\end{array}\right] j=1,2, \ldots, m \quad k=1,2, \ldots, K
$$

It is assumed, according to the general concept of the multistage positional game, that at each discrete moment of the time $t_{k}$ the position of the objects $p\left(t_{k}\right)$ is 
known on the own ship. The constraints of the state co-ordinates:

$$
\left\{x_{0}(t), x_{j}(t)\right\} \in P
$$

constitute the navigational constraints, while the steering constraints:

$$
u_{0} \in U_{0}, u_{j} \in U_{j} \quad j=1,2, \ldots, m
$$

take into consideration the kinematics of the ships' movement, the recommendations of the COLREG Rules and the condition to maintain the safe passing distance $D_{s}$ :

$$
D_{\min }^{j}=\min D_{j}(t) \geq D_{s}
$$

The closed sets $U_{0}$ and $U_{j}$ defined as the sets of the acceptable strategies of the players:

$$
U_{0}[p(t)], U_{j}[p(t)]
$$

are dependent, which means that the choice of the steering $u_{j}$ by the $j$-th object alter the sets of the acceptable strategies of the other objects. Let refer to the set of the acceptable strategies of the own ship while passing the $j$-th encountered object at a distance $D_{s}$. The area, when maintaining stability in time of the course and speed of the own ship and the ship encountered is static and is comprised within the semicircle of a radius equal to the set reference speed of the own ship $V_{r}$ within the arrangement of the co-ordinates $0^{\prime} \mathrm{X}^{\prime} \mathrm{Y}$ ' with the axis $\mathrm{X}^{\prime}$ directed to the direction of the set course, fig. 3 .

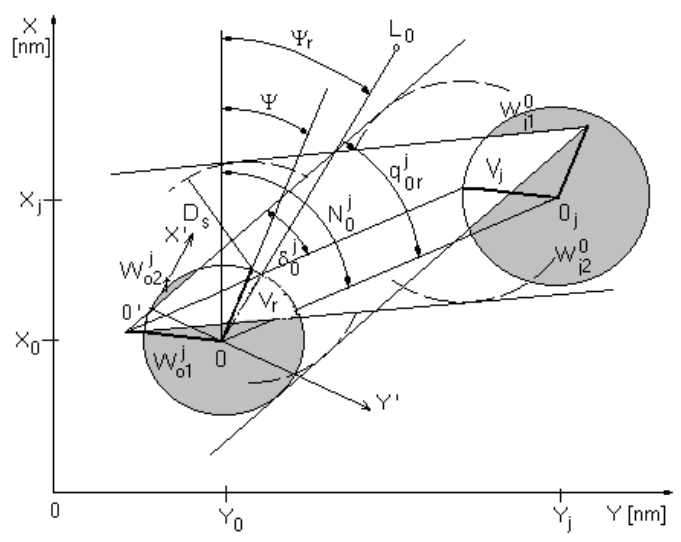

Figure 3: Determination of the acceptable strategies of the own ship and the object encountered. 
The area $U_{0}^{j}$ is determined with the inequalities (8), (9) and (10):

$$
\begin{aligned}
& a_{0}^{j} u_{0}^{x}+b_{0}^{j} u_{0}^{y} \leq c_{0}^{j} \\
& \left(u_{0}^{x}\right)^{2}+\left(u_{0}^{y}\right)^{2} \leq V_{r}^{2}
\end{aligned}
$$

where:

$$
\begin{aligned}
& \vec{V}_{r}=\vec{V}_{0}=\vec{u}_{0}^{2}\left(u_{0}^{x}, u_{0}^{y}\right) \\
& a_{0}^{j}=-\chi_{0}^{j} \cos \left(q_{0 r}^{j}+\chi_{0}^{j} \delta_{0}^{j}\right) \\
& b_{0}^{j}=\chi_{0}^{j} \sin \left(q_{0 r}^{j}+\chi_{0}^{j} \delta_{0}^{j}\right) \\
& c_{0}^{j}=-\chi_{0}^{j}\left[V_{j} \sin \left(q_{j}^{0}+\chi_{0}^{j} \delta_{0}^{j}\right)+V_{r} \cos \left(q_{0 r}^{j}+\chi_{0}^{j} \delta_{0}^{j}\right)\right] \\
& \chi_{0}^{j}=\left\{\begin{array}{l}
1 \text { for } W_{01}^{j} \text { (starboard) } \\
\left.-1 \text { for } W_{02}^{j} \text { ( port }\right)
\end{array}\right\}
\end{aligned}
$$

The symbol $\chi_{0}^{j}$ is determined with the use of an appropriate logical function $Z_{j}$ which characterises particular COLREG Rules. The form of the function $Z_{j}$ depends on the interpretation of these recommendations in order to consider it in the steering algorithm, however:

$$
Z_{j}=\left\{\begin{array}{l}
1 \text { then } \chi_{0}^{j}=1 \\
0 \text { then } \chi_{0}^{j}=-1
\end{array}\right.
$$

The interpretation of the COLREG Rules in the form of the manoeuvring diagrams developed by A.G. Corbet, S.H. Hollingdale, E.S. Calvert and K.D. Jones makes it possible to formulate the logical function $Z_{j}$ as a semantic interpretation of the legal rules of manoeuvring. Each particular ships passing situation is referred to the appropriate logical variable and its value 1 or 0 :

A - encounter of a ship from bow or from other directions, B - closing or sailing away of the ship, $\mathrm{C}$ - passing the ship behind the stern or ahead of the bow, D closing of the ship from bow or from astern, E - closing of the ship from the starboard side or the port side. By minimising the logical function $Z_{j}$ using the method of Karnaugh's tables we can obtain:

$$
Z_{j}=A \cup \bar{A}(\bar{B} \bar{C} \cup \bar{D} \bar{E})
$$

The resultant area of the acceptable manoeuvres of the own ship in relation to the m objects is:

$$
U_{0}=\bigcap_{j=1}^{m} U_{0}^{j} \quad j=1,2, \ldots, m
$$

and is determined by an arrangement of the inequalities (8) and (9). 
On the other hand, however, the set of the acceptable strategies of the $j$-th object in relation to the own ship $U_{j}^{0}$ is determined by the following inequalities:

$$
\begin{aligned}
& a_{j}^{0} u_{j}^{x}+b_{j}^{0} u_{j}^{y} \leq c_{j}^{0} \\
& \left(u_{j}^{x}\right)^{2}+\left(u_{j}^{y}\right)^{2} \leq V_{j}^{2}
\end{aligned}
$$

where:

$$
\left.\begin{array}{rl}
\vec{V}_{j} & =\vec{u}_{j}\left(u_{j}^{x}, u_{j}^{y}\right) \\
a_{j}^{0} & =-\chi_{j}^{0} \cos \left(q_{j}^{0}+\chi_{j}^{0} \delta_{j}^{0}\right) \\
b_{j}^{0} & =\chi_{j}^{0} \sin \left(q_{j}^{0}+\chi_{j}^{0} \delta_{j}^{0}\right) \\
c_{j}^{0} & =-\chi_{j}^{0} V_{0} \sin \left(q_{0}^{j}+\chi_{j}^{0} \delta_{j}^{0}\right)
\end{array}\right\}
$$

The symbol $\chi_{j}^{0}$ is determined by analogy to the determination of $\chi_{0}^{j}$ with the use of the logical function $Z_{j}$ described by the equation (12).

\section{Algorithm of the multistage positional game}

The optimal steering of the own ship $u_{0}^{*}(t)$, equivalent for the current position $p(t)$ to the optimal positional steering $u_{0}^{*}(p)$, is determined in the following way:

- from the relationship (14) and (15) for the measured position $p\left(t_{k}\right)$ of the steering status at the moment $t_{k}$ sets of the acceptable strategies $U_{j}^{0}\left[p\left(t_{k}\right)\right]$ are determined for the encountered objects in relation to the own ship, and from the relationship (8) and (9) the output sets $U_{0}^{j w}\left[p\left(t_{k}\right)\right]$ of the acceptable strategies of the own ship in relation to each one of the encountered objects,

- a pair of vectors $u_{j}^{m}$ and $u_{0}^{j}$, is determined in relation to each $j$-th object and then the optimal positional strategy of the own ship $u_{0}^{*}(p)$ from the condition:

- when the encountered objects non co-operate

$$
I_{1}^{*}=\min _{u_{0} \in U_{0}=\bigcap_{j=1}^{m} U_{0}^{j}}\left\{\max _{u_{j}^{m} \in U_{j} u_{0}^{j} \in U_{0}^{j}\left(u_{j}\right)} S_{0}\left[x_{0}\left(t_{k}\right), L_{0}\right]\right\}=S_{0}^{*}\left(x_{0}, L_{0}\right), U_{0}^{j} \subset U_{0}^{j w} \mid
$$

- when the encountered objects co-operate 


$$
I_{2}^{*}=\min _{u_{0} \in U_{0}=\bigcap_{j=1}^{m} U_{j}^{j}}\left\{\min _{u_{j}^{m} \in U_{j} u_{0}^{j} \in U_{0}^{j}\left(u_{j}\right)} S_{0}\left[x_{0}\left(t_{k}\right), L_{0}\right]\right\}=S_{0}^{*}\left(x_{0}, L_{0}\right), U_{0}^{j} \subset U_{0}^{j w} \mid
$$

- for the non-game optimal control

$$
I_{3}^{*}=\min _{u_{0} \in U_{0}=\bigcap_{j=1}^{m} U_{0}^{j}}\left\{S_{0}\left[x_{0}\left(t_{k}\right), L_{0}\right]\right\}=S_{0}^{*}\left(x_{0}, L_{0}\right), U_{0}^{j} \subset U_{0}^{j w}, j=1,2, \ldots, m
$$

where:

$$
S_{0}\left[x_{0}(t), L_{0}\right]=\int_{t_{0}}^{t_{L_{0}}} u_{0}(t) d t+r_{0}\left(t_{K}\right)+d\left(t_{K}\right)
$$

refers to the goal control function of the own ship in the form of the payments the integral payment and the final one.

The integral payment determines the distance of the own ship to the nearest turning point $L_{0}$ on the assumed route of the voyage and the final one determines the risk of collision and final game trajectory deflection from reference trajectory.

The criteria for the selection of the optimal trajectory of the own ship is reduced to the determination of her course and speed which ensure the smallest losses of way for the safe passing of the encountered objects at a distance not smaller than the assumed value $D_{s}$, having regard to the ship's dynamic in the form of the advance time to the manoeuvre $t_{m}$. The smallest losses of way are achieved for the maximum projection of the speed vector of the own ship on the direction of the assumed course leading to the nearest turning point $L_{0}$.

The optimal steering of the own ship is calculated at each discrete stage of the ship's movement by applying the SIMPLEX method to solve the problem of the linear programming, assuming the relationship (20) as the goal function and the constraints are obtained by including the arrangement of the inequalities (8) and (9) or (14) and (15).

The above problem is then reduced to the following operations: we have to determine the function of the control goal as the maximum of the projection of the speed vector of the own ship on the assumed direction of the movement:

$$
\max \left[F\left(x_{1}, y_{1}\right)=x_{1}\right]
$$

with linear constraints approximating the joint set of the safe strategies of the own ship $U_{0}^{j}$ : 


$$
\left.\begin{array}{l}
a_{0}^{1} \cdot x_{1}+b_{0}^{1} \cdot y_{1} \leq c_{0}^{1} \\
a_{0}^{j} \cdot x_{1}+b_{0}^{j} \cdot y_{1} \leq c_{0}^{j} \\
a_{0}^{m} \cdot x_{1}+b_{0}^{m} \cdot y_{1} \leq c_{0}^{m} \\
a_{0}^{m+1} \cdot x_{1}+b_{0}^{m+1} \cdot y_{1} \leq c_{0}^{m+1} \\
a_{0}^{m+l+s} \cdot x_{1}+b_{0}^{m+l+s} \cdot y_{1} \leq c_{0}^{m+l+s}
\end{array}\right\}
$$

The above constraints are determined by the objects monitored by the ARPA system; this refers to the first $m$ constraints, the next $l$ describe the coastline and the remaining inequalities $s$ approximate the circle of a radius equal to the speed of the own ship. After the interval of time $t_{k}$ the current fixing of the ship's position is carried out and then comes the solving of the problem using the algorithm for the positional steering.

Using the function of $\mathrm{lp}$ - linear programming from the Optimization Toolbox contained in the Matlab, the positional multistage game manoeuvring programs: POSGAME 1 for criterion (17), POSGAME 2 for criterion (18) and POSGAME 3 for criterion (19) has been designed for the determination of the safe ship's trajectory in a collision situation [9].

\section{Computer simulation}

Simulation tests of the POSGAME 1, POSGAME 2 and POSGAME 3 programs have been carried out with reference to real situation recorded on the basis of the radar screen. Fig. 4 shows the computer simulation, performed on the POSGAME programs, for the own ship's trajectory in a situation of passing 42 encountered objects in the English Channel region.

\section{Conclusion}

The application of the model of a multistage positional game for the synthesis of an optimal manoeuvring makes it possible to determine the safe game trajectory of the own ship in situations when she passes a greater number of the encountered objects. The trajectory has been described as a certain sequence of manoeuvres with the course and speed.

The positional game manoeuvring POSGAME computer program designed in the Matlab language also takes into consideration the following: Rules of the Convention on the International Regulations for Preventing Collisions at Sea COLREG, advance time for a manoeuvre $t_{m}$ calculated with regard to the ship's dynamic features and the assessment of the final deflection $d\left(t_{K}\right)$ between the real and reference trajectories.

The big influence for determination of the safe trajectory has the values of time digitization $\Delta t_{k}$ and advance time to the manoeuvre $t_{m}$.

The presented model and method of steering a ship may be used in practice both to improve the shipboard anti-collision system and to construct appropriate ARPA training simulator at Officer Training Centre. 
Good visibility, $\mathrm{D}_{\mathrm{s}}=0.6 \mathrm{~nm}$

Restricted visibility, $\mathrm{D}_{\mathrm{s}}=3.0 \mathrm{~nm}$

$\mathrm{r}_{0}\left(\mathrm{t}_{\mathrm{K}}\right)=0, \mathrm{~d}\left(\mathrm{t}_{\mathrm{K}}\right)=4.67 \mathrm{~nm} \quad$ POSGAME $1 \quad \mathrm{r}_{0}\left(\mathrm{t}_{\mathrm{K}}\right)=0, \mathrm{~d}\left(\mathrm{t}_{\mathrm{K}}\right)=12.11 \mathrm{~nm}$
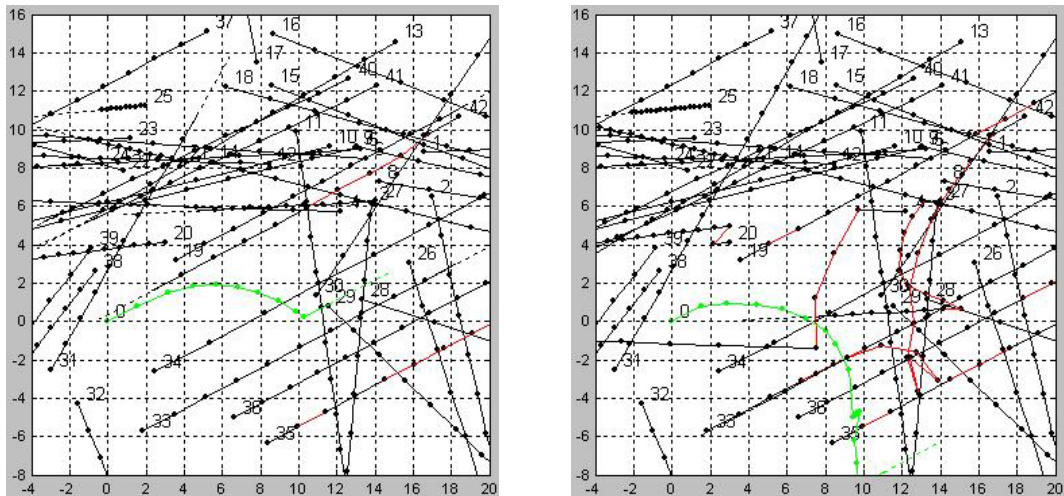

$\mathrm{r}_{0}\left(\mathrm{t}_{\mathrm{K}}\right)=0, \mathrm{~d}\left(\mathrm{t}_{\mathrm{K}}\right)=1.67 \mathrm{~nm} \quad$ POSGAME $2 \quad \mathrm{r}_{0}\left(\mathrm{t}_{\mathrm{K}}\right)=0, \mathrm{~d}\left(\mathrm{t}_{\mathrm{K}}\right)=3.16 \mathrm{~nm}$
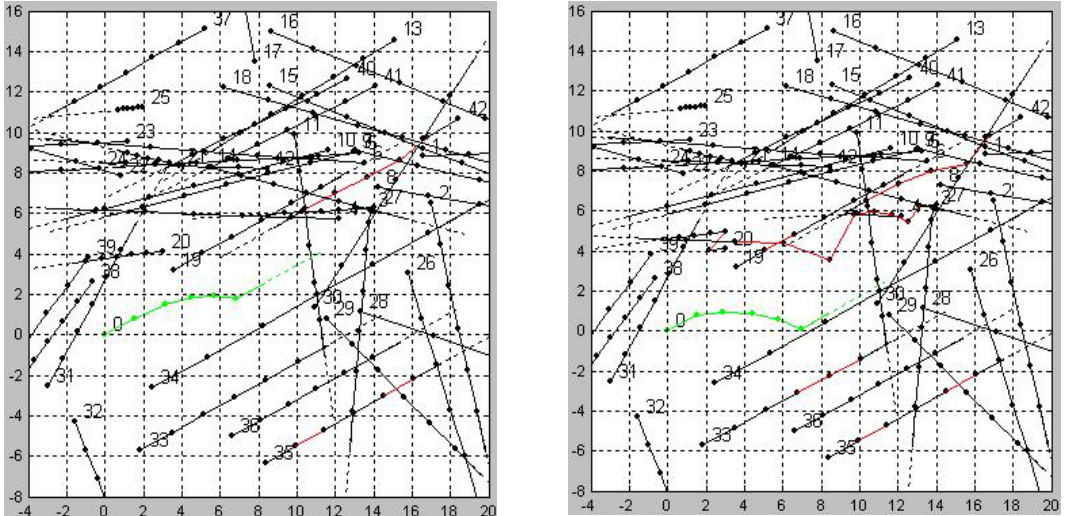

$\mathrm{r}_{0}\left(\mathrm{t}_{\mathrm{K}}\right)=0, \mathrm{~d}\left(\mathrm{t}_{\mathrm{K}}\right)=1.97 \mathrm{~nm} \quad$ POSGAME $3 \quad \mathrm{r}_{0}\left(\mathrm{t}_{\mathrm{K}}\right)=0, \mathrm{~d}\left(\mathrm{t}_{\mathrm{K}}\right)=2.25 \mathrm{~nm}$
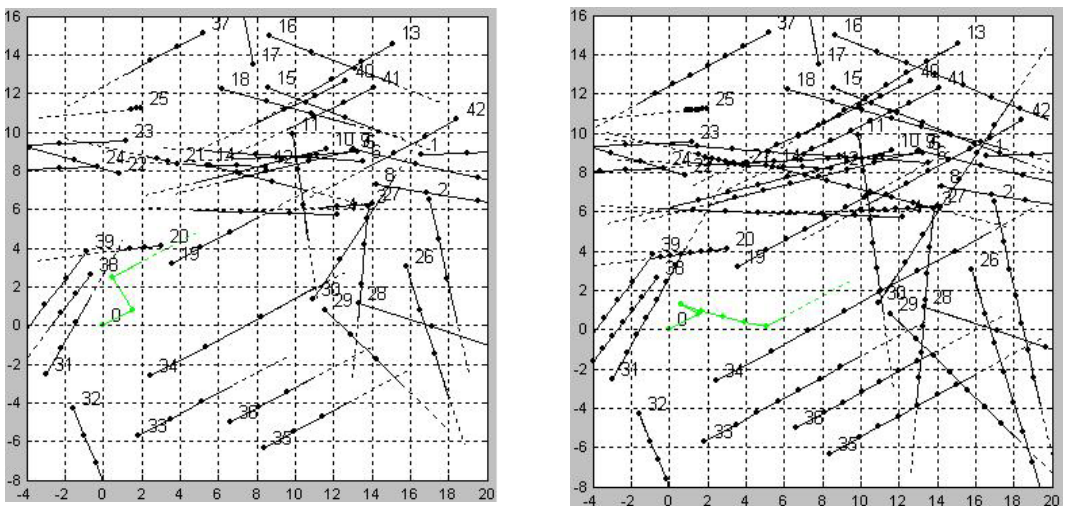

Figure 4: The results of the computer simulation for the safe manoeuvring of the own ship in a situation of passing 42 encountered objects. 


\section{References}

[1] Isaacs R., Differential games, John Wiley and Sons: New York, 1965.

[2] Lisowski J., Mohamed-Seghir M., The safe ship control with minimum risk of collision, Risk Analysis I, Wit Press, Computational Mechanics Publications: Southampton and Boston, pp. 125-134, 1998.

[3] Lisowski J., Simulation models of the safe ship's steering process in collision situations, Risk Analysis II, Wit Press, Computational Mechanics Publications: Southampton and Boston, pp. 13-22, 2000.

[4] Lisowski J., Game control of moving objects. Proc. of the $15^{\text {th }}$ IFAC World Congress: Barcelona, pp.1-6, 2002.

[5] Lisowski J., Multi-step matrix game with the risk of ship collision, Risk Analysis IV, Wit Press, Computational Mechanics Publications: Southampton and Boston, pp. 669-680, 2004.

[6] Lisowski J., The multistage positional game of process decision in marine collision situations. Proc. of the XV International Conference on Systems Science: Wroclaw, pp. 1-10, 2004.

[7] Lisowski J., Mathematical modeling of a safe ship optimal control process. Polish Journal of Environmental Studies, 14(1), pp. 68-75, 2005.

[8] Lisowski J., Game and computational intelligence decision making algorithms for avoiding collision at sea. Proc. of the IEEE Int. Conf. on Technologies for Homeland Security and Safety: Gdansk, pp. 71-78, 2005.

[9] Łebkowski A., Study and computer simulation of game positional control algorithm with consideration ship's speed changes in Matlab: M.Sc. thesis, Gdynia Maritime Academy, 2001 (in Polish).

[10] Merz A.W. \& Karmarkar J.S., Collision avoidance systems and optimal turn manoeuvres. Journal of Navigation, 29(2), pp.160-173, 1976.

[11] Miloh T. \& Sharma S.D., Maritime collision avoidance as a differential game. Schifftechnik, 24(1), pp. 69-88, 1977.

[12] Olsder G.J. \& Walter J.L. A differential game of approach to collision avoidance of ships. Proc. of the $8^{\text {th }}$ IFIP Symposium on Optimization Techniques, pp. 264-271, 1977.

[13] Vincent T.L., Collision avoidance at sea. Proc. of the Workshop Enschede Differential Games and Applications, 1977. 